\title{
Meriania horrida (Melastomataceae), una Especie Nueva de Bolivia
}

\author{
Carmen Ulloa Ulloa \\ Missouri Botanical Garden, P.O. Box 299, St. Louis, Missouri 63166-0299, U.S.A. \\ carmen.ulloa@mobot.org
}

Serena Achá

Herbario Nacional de Bolivia (LPB), Instituto de Ecología, Cota Cota, Calle 27, Campus

Universitario, Casilla 10077, La Paz, Bolivia, y Missouri Botanical Garden, P.O. Box 299, St. Louis, Missouri 63166-0299, U.S.A.

\begin{abstract}
Resumen. Se describe e ilustra una especie nueva, Meriania horrida C. Ulloa \& Achá (Melastomataceae), del departamento de La Paz, Bolivia. Esta especie se caracteriza por tener los tallos, pecíolos y ejes de las inflorescencias cubiertos por un indumento denso de conspicuos tricomas ásperos dendríticos; el ovario tiene lóbulos apicales carnosos y coloridos. Algunas hojas presentan acarodomacios basilaminares. Sólo se conoce de los bosques yungueños en los Andes bolivianos.
\end{abstract}

Abstract. A new species, Meriania horrida C. Ulloa \& Achá (Melastomataceae), from the department of La Paz, Bolivia, is described and illustrated. This species is characterized by the stems, petioles, and axes of the inflorescences covered by a dense indumentum consisting of bristly dendritic trichomes, and by the ovary that presents apical fleshy colorful lobes. Some leaves present acarodomatia at the base of the blades. It is only known from the Yungas forests in the Bolivian Andes.

Key words: Acarodomatia, Andes, Bolivia, IUCN Red List, Melastomataceae, Meriania.

Entre el material colectado en el 2007 dentro del Parque Nacional Madidi en Bolivia se encuentra una llamativa especie nueva en el género Meriania Sw. (Melastomataceae) que describimos a continuación. Meriania se distribuye desde el sur de México (Chiapas) hasta el sureste de Brasil y en las Antillas; es el género más grande de la tribu Merianieae con alrededor de 78 especies (Almeda, 1993; Renner, 1993; Ulloa Ulloa \& Homeier, 2008); en Bolivia se encuentran otras siete especies (Renner, com. pers.).

Meriania horrida C. Ulloa \& Achá, sp. nov. TIPO: Bolivia. La Paz: Franz Tamayo, Parque Nac. Madidi, Keara, Ichucorpa, $14^{\circ} 38^{\prime} 33^{\prime \prime} \mathrm{S}$, $68^{\circ} 57^{\prime} 33^{\prime \prime} \mathrm{W}, 2920 \mathrm{~m}, 4$ nov. 2007, A. Araujo-
Murakami \& N. Chapi 3961 (holotipo, LPB; isotipos, BOLV, K, MO, NY). Figuras 1, 2.

Haec species ab omnibus congeneris indumento denso ex trichomatibus dendriticis asperis conspicuis usque ad $12 \mathrm{~mm}$ longis constante ad caules petiolos et inflorescentiarum rhachides differt.

Arbustos de hasta $3 \mathrm{~m}$; indumento de varios tipos de tricomas: el principal consiste de tricomas ásperos, dendríticos, con aspecto de musgos, con un estípite bien desarrollado, ancho a delgado, de un rosado obscuro y con varios brazos cortos y delgados, verdosos; entremezclados se encuentran tricomas ásperos no ramificados, rojizos; tricomas microscópicos dendríticos, sésiles, con brazos gruesos, amarillento claros; y tricomas microscópicos cortos, simples, rojizos; tallos cuadrangulares a rectangulares, 4-5 × 7-9 mm, engrosados en los nudos, densamente cubiertos por conspicuos tricomas dendríticos de hasta $12 \mathrm{~mm}$ y con tricomas microscópicos esparcidos, glabrescentes. Pecíolos 3-7.5 $\mathrm{cm} \times 1.5-$ $2.3 \mathrm{~mm}$, teretes, algunas veces acanalados en la mitad superior por el lado adaxial, densamente cubiertos con tricomas dendríticos y tricomas no ramificados de 2-5.5 mm y con tricomas microscópicos simples y dendríticos entremezclados y dispersos, glabrescentes; líneas interpeciolares muy delgadas visibles en las ramas glabrescentes; hojas isomorfas, la lámina elíptica, anchamente elíptica a ligeramente obovada, 16-27 × 7.5-14 cm, membranácea, el ápice agudo, la base atenuada, el margen menudamente dentado, los nervios principales 5 , marcadamente triplinerves a 10-23 mm de la base y con un par de nervios suprabasal a $5-13 \mathrm{~mm}$ de la base, rosado oscuros, la nervadura impresa en el haz, muy prominente en el envés, las secundarias separadas $3-5.5 \mathrm{~mm}$, los nérvulos reticulados, el haz glabra, el envés con la superficie cubierta de tricomas microscópicos simples y dendríticos y las venas principales con tricomas similares a los del pecíolo, densos en la base y 


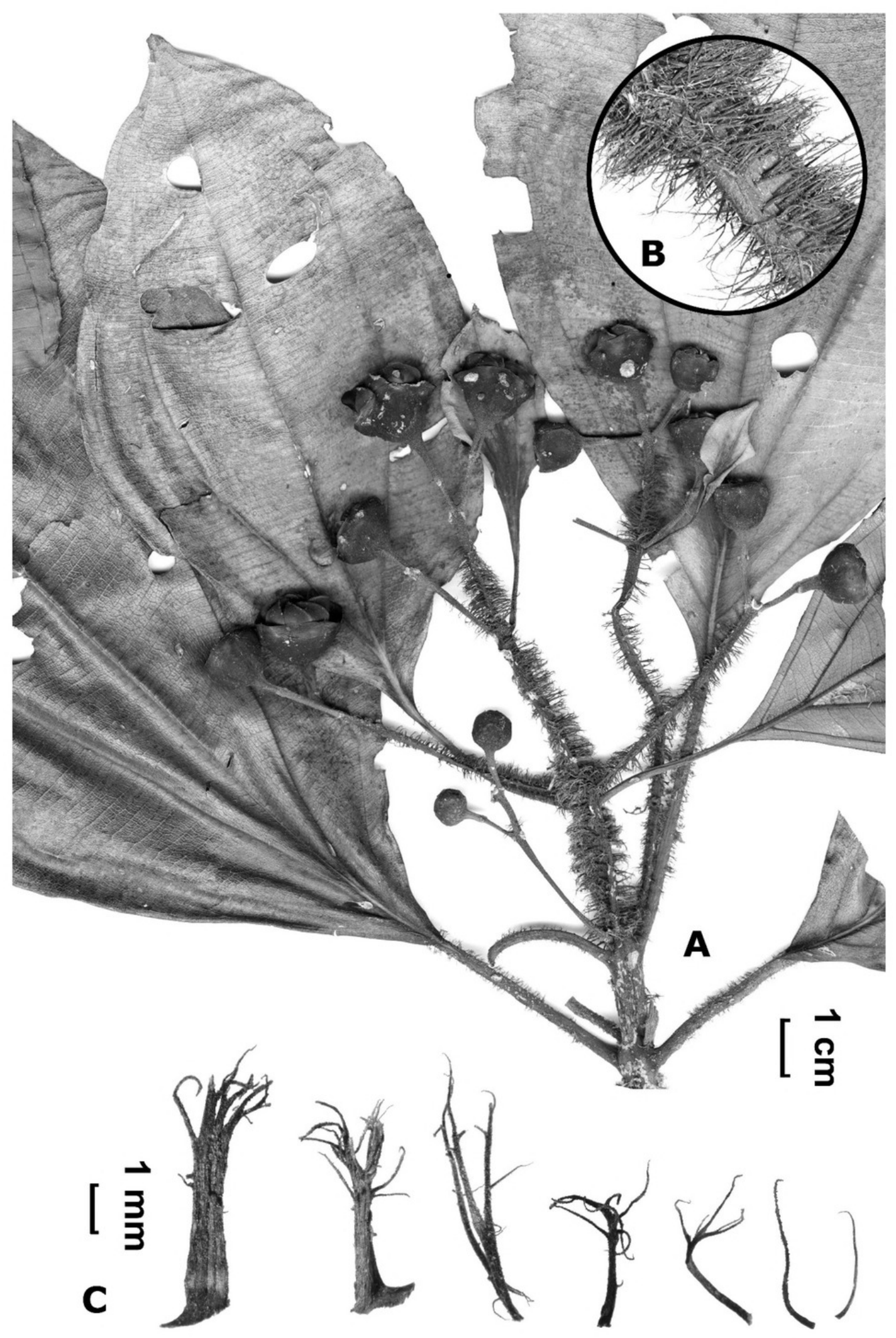

Figura 1. Meriania horrida C. Ulloa \& Achá. —A. Inflorescencia. —B. Detalle del indumento de un eje de la inflorescencia. -C. Tipos de tricomas ásperos individuales (de izquierda a derecha) 1-5, tricomas dendríticos; 6-7 tricomas no ramificados; tricomas que se encuentran en los tallos $(1,2)$, en las inflorescencias (2 a 6), en los pecíolos y las nervaduras de las hojas (5 a 7). A-C, Araujo-Murakami \& Chapi 3957 (MO). 

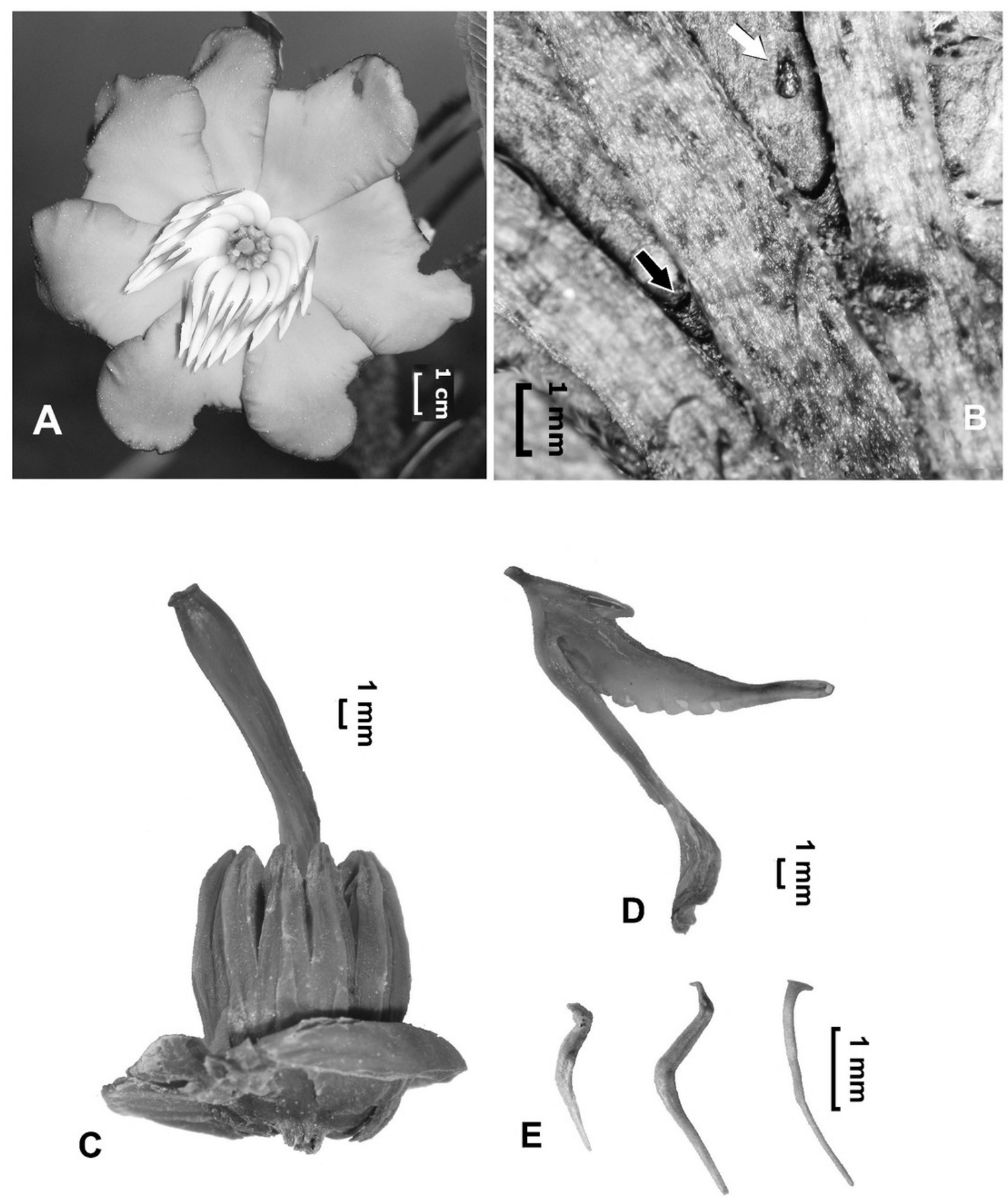

Figura 2. Meriania horrida C. Ulloa \& Achá. - A. Flor en antesis. -B. Acarodomacios en las axilas entre el nervio principal y el primer par de nervios secundarios (flecha negra); se removieron algunos tricomas. La flecha blanca indica un ácaro. - C. Pistilo sobre el hipanto. - D. Estambre vista lateral. —E. Semillas. A, fotografía cortesía A. Araujo-Murakami; B, Araujo-Murakami \& Chapi 3957 (MO); C y D, Araujo-Murakami \& Chapi 3961 (MO, isotipo); E, Fuentes 11846 (MO).

disminuyendo hacia el ápice; acarodomacios presentes a manera de diminutas bolsas de tejido (observados en pocas hojas) entre el nervio principal y el par principal de nervios secundarios por el envés. Inflorescencia un tirsoide compuesto, $11-20 \mathrm{~cm}$ (incluido el pedúnculo), con un par de brácteas foliáceas basales, 5.5-17 × 2.5-9 cm, iguales a las hojas pero más pequeñas, a veces otros pares de brácteas foliáceas más pequeñas presentes más arriba en la inflorescencia, los ejes principales densamente cubiertos por tricomas dendríticos estipitados, de hasta $5 \mathrm{~mm}$, las ramificaciones de último orden con muy escasos tricomas dendríticos y con tricomas microscópicos esparcidos; tirsoide formado por tríades 
de flores, en total hasta 36 flores; bractéolas muy tempranamente caducas (no observadas), dejando una cicatriz notoria. Flores 6(7)-meras, glabras; pedicelos 6-13 mm, rosado oscuros, microscópicamente furfuráceos; hipanto y pétalos de un morado obscuro casi negro brillante (flores cerradas); hipanto campanulado, 8-11 mm, terete, con verrugas dispersas y microscópicamente furfuráceo en la base, coriáceo; toro ca. $1 \mathrm{~mm}$ de ancho, coriáceo; tubo del cáliz terete, ca. $3 \mathrm{~mm}$, los lóbulos del cáliz ovado-deprimidos, ca. $1.5 \times 9 \mathrm{~mm}$, mal definidos, ondulados, morado oscuros, los dientes exteriores del cáliz a manera de muñones en el centro de cada lóbulo calicino, 1$1.8 \mathrm{~mm}$ de diám., café obscuros; pétalos contortos, obovados, 30-40 × 30-35 mm, estrechos hacia la base, oblicuamente redondeados en el ápice, gruesos, patentes y ligeramente convexos en la floración, rosado intenso brillantes, apicalmente ciliados en las flores cerradas; estambres isomorfos, 12, 13 o 14, todos dirigidos hacia abajo en forma de abanico, geniculados en el punto de inserción del filamento, glabros; filamentos 9-12 mm, blanco brillantes con tintes rosados, complanados (una de la flores presenta fusión de dos filamentos), las tecas marcadamente subuladas, $8.5-10 \mathrm{~mm}$, rosado-violáceas de la mitad hacia el poro, blancas hacia la inserción con el filamento, el poro apical dorsalmente inclinado, el conectivo no prolongado bajo las tecas, dilatado dorsalmente, 6.5-7 mm, blanco, con un apéndice subulado de ca. $1 \mathrm{~mm}$, proyectado hacia afuera, con la punta rosada y un apéndice oblongo y romo, ca. $2 \mathrm{~mm}$, proyectado hacia adentro; ovario 6-locular, libre, 8$10 \mathrm{~mm}$ de alto (incluidos los lóbulos), carnoso, conspicuamente acostillado, cada carpelo bilobulado en el ápice y prolongado $3.5-5 \mathrm{~mm}$ sobre la base del estilo, los lóbulos triangulares, agudos, separados en el ápice, rosado intensos, el estilo rosado intenso, terete, $9-15 \times 1.2-1.5 \mathrm{~mm}$, ligeramente declinado y curvado en el ápice, el estigma truncado, blanco, la placenta axilar, con forma de yunque, ocupando toda la cavidad del lóculo, los óvulos diminutos, alargados, numerosos. Frutos capsulares, 1.2-1.7 × 1.3-2.5 cm, más anchos que altos, loculicidas, con las valvas papiráceas persistentes, el hipanto persistente, cubriendo la base, los frutos viejos con restos fibrosos de las paredes; semillas muy numerosas, $2-4 \mathrm{~mm}$, largamente cuneadas, arqueadas, deflexas, algunas con forma de $\mathrm{S}$ alargada, estrechas, lisas y brillantes.

Distribución, hábitat, fenología y conservación. Esta especie se conoce únicamente de tres localidades en bosques montanos yungueños dentro del Parque Nacional Madidi en el departamento de La Paz, provincia de Franz Tamayo, entre 2300 y 2920 m, en la vertiente oriental de los Andes bolivianos; crece junto a Clethra cuneata Rusby, Hesperomeles ferruginea (Pers.) Benth., Weinmannia fagaroides Kunth, Myrsine dependens (Ruiz \& Pav.) Spreng. y especies de Miconia Ruiz \& Pav. y Clusia L. Ha sido recolectada con flores en noviembre y con frutos en mayo y noviembre. El acceso al área donde crece esta especie es por caminos de mula y si bien existen trazos de carreteros para planes futuros, dudosamente afectarán a las poblaciones de esta especie que crecen en filos escarpados (A. Fuentes, com. pers.).

El área de ocupación (AOO) es de $27 \mathrm{~km}^{2}$ (celdas de $3 \times 3 \mathrm{~km}^{2}$ ) y su extensión de ocurrencia (EOO) es de $4.1 \mathrm{~km}^{2}$, con el $0 \%$ de disminución futura (ya que $100 \%$ se encuentra dentro de un área protegida o área prístina). De acuerdo al conocimiento actual que tenemos para esta especie la categorización de amenaza según los criterios de la IUCN (2001) es de Preocupación Menor (LC) sobre la base del criterio B de tamaño del área de distribución geográfica (IUCN Standards and Petitions Working Group, 2008).

Etimología. El epíteto específico se refiere al denso indumento de tricomas ásperos (horridus), largos y ramificados que cubren los tallos, pecíolos y ejes de la inflorescencia.

Discusión. Meriania horrida se distingue de todas las especies del género por los conspicuos tricomas dendríticos que cubren los tallos, pecíolos y ejes de las inflorescencias. Dentro del género Centronia D. Don, género dudosamente distinto (actualmente bajo revisión por H. Mendoza, Bogotá, véase Triana [1871], Wurdack [1973], Almeda [1993], Schulman \& Hyvönene [2003] acerca de la separación de este género), C. mutisii (Bonpl.) Triana presenta un indumento denso de tricomas largos, de hasta $5 \mathrm{~mm}$ que cubre los tallos, pecíolos, ejes de la inflorescencia, pedicelos y hasta la mitad del hipanto y entremezclados otros tricomas cortos, gruesos; ambas especies tienen estambres con proyecciones del conectivo similares y el ovario presenta lóbulos apicales, siendo los de C. mutisii más divididos. Sin embargo, en C. mutisii los tricomas largos son enteros, la especie es marcadamente distinta por las flores caliptradas la yema y los pétalos que se tornan azulados, las hojas son peltadas, acorazonadas y 7 nervias.

Dentro de las especies actualmente bajo Meriania algunas tienen notorio indumento, e.g., M. tomentosa (Cogn.) Wurdack, M. macrophylla (Benth.) Triana, M. versicolor L. Uribe, M. weberbaueri J. F. Macbr., pero éste no sobrepasa los $2 \mathrm{~mm}$ y los caracteres no son comparables con la especie nueva.

Meriania horrida se parece a M. hexamera Sprague, una especie ampliamente distribuida desde el sur de Colombia hasta el este de Perú entre 1100 y 
2000(2200) m. Ambas especies tienen las inflorescencias robustas de tamaño y arreglo similares, con las brácteas foliáceas y bractéolas tempranamente caducas, los pedicelos robustos, las flores 6-meras de color magenta, el hipanto carnoso, los dientes exteriores del cáliz a manera de muñones, los pétalos de tamaño similar, los estambres isómeros, dirigidos hacia abajo en la flor, el ovario libre, carnoso con carpelos bilobulados en el ápice, las cápsulas están rodeadas por el hipanto en la base. Sin embargo, $M$. hexamera es una planta prácticamente glabra con indumento microscópico, las hojas son la mitad del tamaño $(9-15 \times 3.5-14 \mathrm{~cm})$, cortamente acuminadas en el ápice, el margen entero, algunas veces revoluto en la base; el conectivo dilatado no presenta el apéndice romo proyectado hacia adentro y las anteras son tricolores, rosado-violáceas hacia el poro, blancas en el centro y amarillo intenso hacia la base dorso basal del conectivo, mientras en la nueva especie son bicolores, rosado-violáceas hacia el poro y blancas hacia la base; los lóbulos del ovario son redondeados mientras que son triangulares y agudos en la nueva especie. Las semillas de $M$. hexamera son largamente cuneadas, de hasta $2 \mathrm{~mm}$, rectas.

Ambas especies presentan acarodomacios basilaminares por el envés de las hojas a manera de bolsas de tejido en la unión de los nervios. En Meriania hexamera, los domacios son bastante obvios en la unión de los tres nervios principales, donde se forman pequeñas bolsas huecas o se presentan a manera de una extensión incurvada de tejido basilateral a ambos lados del nervio principal. En M. horrida los domacios no se observaron sino en pocas hojas y son en forma de pequeñas bolsas en la unión de los nervios, se documenta también la presencia de diminutos ácaros (Fig. 2B). Si bien la presencia de tejido en la unión de los nervios no es rara en otros géneros de la familia, en Meriania se ha documentado en pocas especies (e.g., Goldenberg \& Martins, 1999).

Paratipos. BOLIVIA. La Paz: Franz Tamayo, Parque Nac. Madidi, Keara, Ichucorpa, $14^{\circ} 38^{\prime} 33^{\prime \prime} \mathrm{S}, 68^{\circ} 57^{\prime} 33^{\prime \prime} \mathrm{W}, 4$ nov. 2007, A. Araujo-Murakami \& N. Chapi 3957 (AAU, BOLV, CAS, LPB, M, MO, USZ); Parque Nac. Madidi, Mojos,
Tocoaque, por el camino a Queara, $14^{\circ} 37^{\prime} 06^{\prime \prime} \mathrm{S}, 68^{\circ} 57^{\prime} 07^{\prime \prime} \mathrm{W}$, 13 mayo 2007, A. Fuentes 11846 (LPB, MO, NY); Parque Nac. Madidi, sector Santa Ana, por el antiguo camino Pelechuco-Apolo, subiendo cerro al SE del campamento, $14^{\circ} 46^{\prime} 30^{\prime \prime} \mathrm{S}, 68^{\circ} 58^{\prime} 13^{\prime \prime} \mathrm{W}, 25$ jun. 2009, A. Fuentes, J. Salas \& R. Huasurco 14831 (BOLV, COL, LPB, MO, QCA).

Agradecimientos. Agradecemos a R. Gereau por traducir la diagnosis al latín y T. Distler, V. Torrez y A. Fuentes por su ayuda en relación a la clasificación de amenaza. F. Keusenkothen escaneó los ejemplares de herbario; F. Michelangeli nos facilitó detalles fotográficos de material depositado en NY; y A. Araujo-Murakami nos envío la fotografía de campo. Agradecemos los comentarios de dos revisores anónimos.

\section{Literatura Citada}

Almeda, F. 1993. An evaluation of the Mesoamerican species of Meriania (Melastomataceae: Merianieae). Proc. Calif. Acad. Sci., ser. 4, 48(7): 141-152.

Goldenberg, R. \& A. B. Martins. 1999. Two new Melastomataceae from São Paulo, Brazil. Kew Bull. 54: 465-470.

IUCN. 2001. IUCN Red List Categories and Criteria, Version 3.1. Prepared by the IUCN Species Survival Commission. IUCN, Gland, Switzerland, and Cambridge, United Kingdom.

IUCN Standards and Petitions Working Group. 2008. Guidelines for Using the IUCN Red List Categories and Criteria, Version 7.0. Prepared by the Standards and Petitions Working Group of the IUCN SSC Biodiversity Assessments Sub-Committee in August 2008. Downloadable from < http://intranet.iucn.org/webfiles/doc/SSC/RedList/RedListGuidelines.pdf $>$.

Renner, S. S. 1993. Phylogeny and classification of the Melastomataceae and Memecylaceae. Nordic J. Bot. 13: 519-540.

Schulman, L. \& J. Hyvönene. 2003. A cladistic analysis of Adelobotrys (Melastomataceae) based on morphology, with notes on generic limits within the tribe Merianieae. Syst. Bot. 28: 738-756.

Triana, J. 1871. Les Mélastomatacées. Trans. Linn. Soc. London 28: 1-188.

Ulloa Ulloa, C. \& J. Homeier. 2008. Meriania franciscana (Melastomataceae), una especie nueva de los Andes de Ecuador. Anales Jard. Bot. Madrid 65: 383-387.

Wurdack, J. J. 1973. Melastomataceae. Pp. 178-188 in T. Lasser (editor), Flora de Venezuela, Vol. 8(1). Instituto Botánico, Ministerio de Agricultura y Cría, Caracas. 


\section{$2 \mathrm{BHL}$ Biodiversity Heritage Library}

Ulloa Ulloa, Carmen and Achá, Serena. 2010. "Meriania horrida (Melastomataceae), una Especie Nueva de Bolivia." Novon a journal of botanical nomenclature from the Missouri Botanical Garden 20, 371-375.

View This Item Online: https://www.biodiversitylibrary.org/item/123332

Permalink: https://www.biodiversitylibrary.org/partpdf/122042

\section{Holding Institution}

Missouri Botanical Garden, Peter H. Raven Library

\section{Sponsored by}

Missouri Botanical Garden

\section{Copyright \& Reuse}

Copyright Status: Permission to digitize granted by rights holder Rights: https://www.biodiversitylibrary.org/permissions

This document was created from content at the Biodiversity Heritage Library, the world's largest open access digital library for biodiversity literature and archives. Visit BHL at https://www.biodiversitylibrary.org. 